\title{
PROGRESSIVE MANAGEMENT METHODOLOGY FOR REAL-TIME BUSINESS INTELLIGENCE DECISION SYSTEMS
}

\author{
Cristian PĂUNA \\ Economic Informatics Doctoral School \\ Bucharest University of Economic Studies, Romania \\ cristian.pauna@ie.ase.ro
}

\begin{abstract}
Business intelligence systems represent a significant trend today. Choosing the right project management methodology is an essential step for a successful business intelligence implementation. New aspects and perspectives are included in this process nowadays due to new requirements imposed by the real-time activities. The automated decision-making systems used in different activity domains and the low-latency responses required by different processes determine new specifications for the entire system. The response delay of each time chain component has become a design factor. Also, using automated decision-making systems, the human factor is excluded from an important part of the decision process. To manage the decision tree appropriately, the human and automated decisions units must also be included in the business intelligence system design. It was found that the results obtained after the implementation of a real-time decision system will conduct to new requirements for the business intelligence system itself and will produce new resources for a better and improved solution. This progressive implementation needs a suitable management methodology in order to permit evaluative adaptability for the entire system. This paper will present the Progressive Management Methodology especially designed for a successful Real-Time Business Intelligence Decision System implementation. The model permits the analysis, design, implementation, and improvement for the real-time components considering the time-delay as a design factor.
\end{abstract}

Keywords: business intelligence system, automated decision-making system, real-time system, low-latency response, decision tree, progressive management methodology.

JEL classification: M15, O16, G23

DOI: $10.12948 / \mathrm{ie} 2019.05 .01$

\section{Introduction}

Design and implementation of business intelligence systems (BIS) is an essential activity today. In order to adapt and improve business decisions, more and more companies are developing complex BIS. "Global revenue in the business intelligence and analytics software market is forecast to reach $\$ 18.3$ billion in 2017 , an increase of 7.3 percent from 2016 , according to the latest forecast from Gartner, Inc. By the end of 2020, the market is forecast to grow to $\$ 22.8$ billion." [1] This fast-growing market still faces challenges, especially due to the economic climate changes and also as a result of continuous modifications in the systems requirements. It is well known that an important part of the BIS fails in their implementation attempt due to inappropriate project management, as a result of the inadequate analysis process, or just because of the misallocation of the resources. From the known predictions, "through 2017 , $60 \%$ of big data projects will fail to go beyond piloting and experimentation, and will be abandoned." [2] This paper aims to improve this factor analyzing different failure reasons and improving the project methodology in order to increase the success rate. The original 
Progressive Management Methodology (ProgressM) will be revealed in this article together with practical examples and facts from a Real-Time Business Intelligence Decision System implemented with this method.

It is well known that the BIS analyze the past in order to improve the future using information technology. These systems are "broad category of applications, technologies, and processes for gathering, storing, accessing and analyzing data to help business users make better decisions." [3] Even they are technological systems, the human factor is involved in this process. The failure or success of a BIS implementation can be quantified after the decisions taken will produce economic effects in the enterprise. It was found that resource planning is a critical activity and it is mandatory to be included in BIS development as a standalone process. Besides, the decision tree must also be included in the BIS design. More, once the system uses automated-decision processes, the decision tree becomes a significant part of the system. The business needs will impose the BIS project requirements. These factors are in constant change today. It was found that a critical failure factor for the BIS is the delay between the data analysis results and the decision support level needs. Informatics systems providing aggregated data and reports one time per day for a decision activity that needs real-time information for quick adjustments of the main activity is not a successful solution. Even an update made one time per hour for the aggregated data will not assure a stable functionality of a real-time decision process. For these cases, real-time business intelligence systems (RTBIS) are required. These are systems using applications, technologies, and processes to collect, access, store and analyze real-time data streams in order to aggregate real-time decisions. Due to the fast speed implied, RTBIS usually include automated decision-making components to assure the fast response required. These are called in this paper Real-Time Business Intelligence Decisions Systems (BIDS); they are BIS generating and executing real-time automated decision streams to produce enterprise results.

A particular domain where BIDS are required is the domain of capital markets. Due to the large price volatility in the financial markets, the trading and investment decisions must be made and executed in a very short time in order to catch the best price levels. The profit is the reason to participate in the capital markets. It is made by the difference between the buy and the selling prices. A fast evolution price market involves real-time price data analysis and a real-time decision process to be organized in order to buy or sell at the desired price. The delayed orders received by the brokerage system will be ignored if the price is too far away from the current real-time quote. All of these conditions describe as a design factor for BIDS for modern financial investment companies: the low-latency response of the entire system.

This paper will present a modern methodology to design, build, implement, maintain, manage and evolve the BIDS: the Progressive Management methodology (ProgressM). The model will include the resource planning methodology and interactive processes in order to design and implement the real-time chain components. It was found that the latency response of each component is a design criterion and special methods are involved in order to manage these systems. The low-latency functionality of all components also conducts to a new architecture, specific design processes, and specific methodology to manage the entire real-time system being used.

\section{Business Intelligence Systems}

The BIS is a system analyzing the past data in order to build decisions to gain a specific result for the future. "The future is informed by the past." [3] The first idea became from 1950 in terms of "Interaction Support Analysis" [4] from a study of small groups about how the resulting data can be analyzed and summarized in order to retrieve useful information. Later in 1960, the concept of "Decision Support Systems" became a new idea due to "the technical 
work on interactive computer systems." [5] Starting with 1970 different business journals has published different papers about "Management Decision Systems and Strategic Planning Systems." [6] These concepts have become in 1980 the well known "Executive Support Systems, owing to the increasingly complicated and volatile environment of decision-making application." [7] "Online Analytical Processes is an approach to answering multi-dimensional analytical" [8] which have evolved continuously in the years of 1990. The BIS term is used today in order to cover all these systems under a unitary project. BIS "architecture is a set of concepts, tools, methods, and technologies that, once connected, create knowledge and meet the strategic needs of the company." [9] BIS is designed to manage the past data in order to report the state of the business to help for a more sustained decision. RTBIS analyze real-time data streams, and the reports are built in real-time, including the present data. The fast-growing real-time data systems development pushes to new limits the streaming data analysis. The realtime analysis became an essential factor in BIS today especially for the finance industry. This fact is confirmed by the BIS survey published by Forbes in [10]. However, analyzing the data streams is not enough for different business nowadays.

\section{Project Management Methodologies}

As we have seen, BIS is a complex system integrating multiple data sources, distributed informatics systems, and multiple data and reporting processes in order to offers sustained information for the decision level. The project management methodology applied to design and implement BIS may vary depending on the business specificity. Over the years many authors have established several types of methods or factors that can be applied for the informatics system particularly or generalized for complex BIS. Some authors use different critical factors to break the design process into sequential phases depending on the specificity of the project. Methodologies including increased management and control steps are developed and presented in [11], [12] or [13]. Other authors [14] indicate as a key factor the communication of all successful practices used and build additional support for inexperienced or unexceptional developers or phases [15]. The project management methodology must build a perspective to solve all challenges during the project implementation. Starting with the first analysis phases, the methodology must respond to all steps including the project and requirements modifications due to economic, functional or environmental fluctuations.

There is sustained research about the formal and strict methodologies for information development systems, particularly during the time. More researches are presented by [16] or [17]. Several authors' present studies about different adaptations of the known methodologies to the specificity of the meaning of the complex project omitting, changing or adding substantial steps as it can be discovered in [18], [19] or [20]. It was found that the BIS involve significative differences during the project implementation compared with the simple informatics systems. The business processes related to the functionality of the entire BIS implies specific flexibility for all the steps during the project management and implementation. In BIS we have a data functionality implementation which demands cross-organizational activities to be developed and planned. In addition, aspect as low-latency response gets special attention. An interesting approach in order to design the development steps is presented in [21]. Being a data-driven process with more flexibility needed in the management process, the BIS project can be managed with an agile methodology but not Scrum which is a code-driven methodology [22]. A significant degree of planning and coordination is needed together with enough flexibility to integrate all changes during the implementation project. No universal methodology can be applied blindly for any BIS system design. More sustained arguments can be found in [23]. 
The specificity of each business must be considered in order to build a suitable situation-based methodology for each BIS and to avoid the risk of using an inadequate model. More considerations about this idea can be found in [24] together with the sustained idea that the decision regarding the methodology used to manage the BIS project is based on the majority of cases on the individual preferences of the designers and project managers than rational aspects, in case there are no specific requirements from the company ownership. In [25] we can find that a sustained investigative approach will improve the BIS methodology to fit the type of the project, better than a classic or prescriptive approach. Under all of these considerations, this paper will reveal a methodology to manage the BIDS.

The flexibility criterion can classify the project management methodologies as we can see in figure 1. In the low flexibility range, we have the fixed plan methodologies as the Waterfall method [26], Cleanroom method [27] or the Capability Maturity Model [28]. With a little higher flexibility we have the iterative models as it is the Spiral model [29]. With more flexibility, there are incremental models as it is the Rational Unified Process [30]. The agile models are those with the highest flexibility level as Scrum [31] or eXtreme Programming Model [32]. Looking at the specificity of each model type we can summarize that the agile methods "count on their rapid iteration cycles to determine needed changes in the desired capability and to fix them in the next iteration. Determining the highest-priority set of requirements to be included in the next iteration is done collaboratively by the customers and developers." [23]. The iterative and incremental methodologies can be adapted to include the changes during the design progress [32] more easily than the planned-based models which are known as the most uncomfortable when it is about the business or requirements changes during the project. Several more considerations can be found in [24]. For the real-time BIS, an adaptable model is needed, but the control is also required in order to measure and manage different characteristics for those process included in the real-time chain.

\section{Progressive Management Methodology}

In this section, it will be presented an original project management methodology which combines the advantages of all known models mentioned in the last chapter with improved factors in order to respond to all requirements of BIDS projects. To add value from the scientific point of view, the methodology presented in this paper must answer to a specific list of arguments. First, the methodology must be applicable to a representative class of problems; this is the BIDS including automated decision-making processes. Second, the method presented must include new findings and new knowledge. These are related to the aspects of adaptable resource management, the real-time data processes, and the model to evaluate and distribute the time delay in the time-chain in order to obtain a low-latency response and how the components of the automated decision can be integrated into the decision tree in order to manage the functionality of the entire system. Third, the methodology presented must have reproducibility and to be applicable to a new case in the same hypothesis. These factors are assured by the generality of the presented model which can be applied for any BIDS regardless of the company's activity. On the fourth scientific point of view, the method must generate value to a class of users or companies. This is a fact; the methodology was already implemented to a BIDS for a capital investment company and can be applied in the future to any other similar enterprise. Besides, the methodology presented can be applied to any other real-time activity from any other domain. The fifth scientific value criterion asks the methodology to be feasible and the costs and application effort to be justified by the obtained benefits. The sixth point of view is to adopt a value-based approach in order to develop and classify the metrics of the presented methodology. 
www.conferenceie.ase.ro

\subsection{ProgressM principles}

In the era of agile methodologies for software and project management, lack of a general plan is a failure factor. One of the key roles of the BIS project manager, BIS architect, BIS master or whatever name can have the responsible job for the BIS success is to manage all the activities and resources in order to obtain functional BIS, and to adapt the whole process to all discovered changes. There are three major factors considered as ProgressM principles:

- Any action must be accompanied by the necessary and sufficient resources;

- The progress in any activity comes from small, multiple, complete and consecutive steps;

- Unplanned interactivity between components must be followed by control keys and points. These factors come from experience in different agile project activities. The ProgressM principles are developed in order to avoid the failure of the implemented project. In practice, it was observed in many cases when the necessary resources were unavailable for a specific requirement or task. This is probably one of the significant failure reasons. A simple example is that real-time BIS cannot work without real-data streams. Alternatively, real-time BIS cannot work properly with only one real-time data supplier. Any interruption in the data stream connection will put the enterprise in a nonfunctional stage. Factors like these are decided in the business analysis and business modeling and here comes the experience of a good project manager to ask for suitable resources form the beginning. An inexperienced manager will drive on the wrong ways from the beginning. Another example about inadequate resource allocation is in a more advanced progress stage when the managers and owners understand that need a specific state of acts, for example, real-time reports and they allocate no additional resources for this new improvement. We can handle what we have sentence is not working any time, and the result can be the failure of the whole BIS.

The second principle integrated into the ProgressM methodology becomes from the reality that usual complex activities or processes do not have an explicit algorithm or solution. The majority of new business and enterprises are trying to be unique in order to gain a competitive advantage. A unique activity supposes a unique or a new idea, an unknown algorithm and an unknown technical way in order to find a unique technical solution. The ProgressM methodology is designed especially for this case when unknown solutions must be found in order to solve the BIS problems. In these cases, the progress and success of any activity come from small, multiple, complete and consecutive steps. Any components of BIS will be designed and implemented in small steps. To accomplish the objective, multiple steps are necessary. Usually, the next step can be initiated after the last objective was achieved. In an agile environment, multiple steps can be consecutively initiated. In a key-driven methodology, the steps will be confirmed as finished consecutively, once the key factor or the desired efficiency level was achieved. Third principle included in the ProgressM methodology is that we can not have a general plan for all actions, more flexibility is needed especially for those unknown steps which make the BIS unique, a high degree of interactivity is necessarily between all components of the system, including the resource components but for each interactivity must be planned a critical factor in order to measure the progress.

\subsection{ProgressM steps}

The ProgressM methodology presented in this paper was applied for the first time to build, implement, maintain and improve BIDS of a financial trading company. We will use this particular example to present the methodology and how it can be applied. The model was designed starting to the initial criteria imposed by the specificity of the mentioned activity:

a). The initial business requirements are known, but because the company activity is new and implies new technologies and techniques with fast time evolution, the business requirements will be changed from time to time. Also, some of the technical solutions are unknown in the 
initial business analysis process. Starting from this idea, the model can not use a plan-driven methodology; a higher degree of flexibility must be involved in order to develop new or unknown business components. The new technologies used can produce new requirements in the decision-making chain which presume new requirements for the entire BIS. Once the new requirements are produced and delivered by the BIS and can be sustained by the new resources also produced by the BIS itself, we will call this as Progressive Management Methodology, on short ProgressM. The logical of this methodology is presented in figure 1.

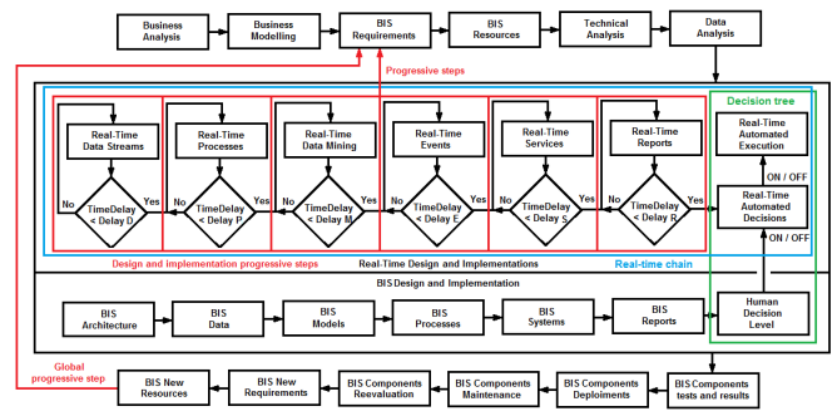

Figure 1. Progressive Management Methodology for Real-Time Business Intelligence Decision Systems.

b). Because it is about BIDS, the design key factor will be the time delay for each BIS components in the time-chain. Into a system with inputs as real-time streams, the extract, transform and load processes must be done with low-latency to deliver fast data for the online analytical processing and services. The time-chain is the sum of all real-time components. The real-time automated decisions are made at the end of the time-chain functionality, and the total delay is also a design factor for the entire BIS. For this purpose, the model will use an iterative cycle for each time-chain component and a sequential design to link all the time-chain components with the other BIS components. This ProgressM method can be applied in any BIDS design when a specific measure is used as a design factor.

c). The methodology must permit a link between the automated decision and the human decision factors in order to integrate all decisions into a decision tree. The human factor decision level will control and manage automated decision processes. They all contribute together to the results of the enterprise. Once the automated decisions are executed by the priory designed automated processes, the implementation of the BIS decision system will produce results, but equally will produce new requirements. This fact will call again the progressive global cycle, and the methodology must answer to the management needs in order to quantify the new requirements, to measure the new resources available and to plan a new progress step, which will be an incremental global process for the whole system.

d). The links between the BIS processes and services and the real-time decision chain actions can also evolve as direct results of the BIS operation. Learning from the past must be an included process in the design methodology. This step becomes a requirement for the project management methodologies in the era of fast technic changes. From this reason, a more flexible methodology is needed for each component, into or outside the time-chain.

e). The human and automated decisions must also be included in the BIS design, and implementation process and a management model for the decision tree has to be inserted in the methodology. Starting from the business analysis, and based on the business models, a finite list of decisions can be built and linked with the functionality of the whole business system. The automated decision processes can be configured by the human decision factors and can also be started or stopped in different moments depending on specific functionality 
requirements. New requirements on the BIS activity will ask for new automated decision processes which will be a sustained design factor for the next progress design step.

Based on the three main principles exposed in the last chapter, with these requirements for the BIDS, the Progressive Management Methodology developed has the logical included in figure 2. The steps to apply the methodology to BIDS are: 1. Business Analysis 2. Business Modeling 3. BIDS Requirements 4. BIDS Resources 5. Technical Analysis 6. Data Analysis 7. System Architecture 8. Real-Time Chain Analysis 9. Real-Time Data Streams 10. Real-Time DataMining 11. Real-Time Processes 12. Real-Time Events 13. Real-Time Services 14. Real-Time Reports 15. Real-Time Automated Decisions 16. Real Time Automated Execution 17. Connections with Real-Time Chain 18. Data Design and Implementation 19. Models Design and Implementation 20. Processes Design and Implementation 21. Systems Design and Implementation 22. Reports Design and Implementation 23. Decision Tree design and Implementation 24. BIDS Components Tests and Results 25. BIDS Components Deployment 26. BIDS Maintenance 27. BIDS Reevaluation 28. BIDS New Requirements 29. BIDS New Resources 30. BIDS Progressive step.

\subsection{ProgressM is more than Agile}

ProgressM is a methodology to develop large scale projects. ProgressM can include an agile methodology for any software components, but the design and implementation process is directed, organized and controlled according to the three fundamental ProgressM principles: any action needs resources, progress comes from small steps, and control key factors must follow all unplanned activities. ProgressM methodology is more than agile. The methodology tries to control the failure factors. ProgressM methodology can also be applied to small projects, even to single software development if all three fundamental principles are met.

According to the Manifesto for Agile Software Development [33], there are twelve agile principles. In short they are related to the priority to satisfy the customer, to accept changing requirements, to deliver software working frequently, business people and developers must work together, build projects around motivated individuals, initiate and sustain face-to-face conversation, working software is the primary measure of progress, promote suitable development maintaining a suitable peace, get attention to technical excellence, simplicity is essential, the best requirements emerge from self-organizing teams and adjust the team behavior to become more productive. These are the agile principles.

It was found that applying the agile principles on large scale projects many failure factors can be inserted by an unrealistic approach or by inappropriate project management. This is what ProgressM tries to correct. It was found that these three fundamental principles of the ProgressM methodology cover a lot of missing points and reduce the failure factor significantly. The first principle assures that any action, new procedures, event, modules, process, service, software or not software component integrated into a project must be accompanied by suitable resources. The principle comes from the fact that action without resources has a substantial probability of failing. Working often into an agile environment the author of this paper met the situation when a new software module comes into the plan after an iterative analysis. The component was not in the plan from the beginning, so there are no resources allocated for it. The project manager asked for the new component to be delivered under the idea: we work into an agile environment, it was not in the initial plan, and we need it, so we have to do it. Is that so? Can be done without any additional resources? At this moment the manager is increasing the failure probability of the project, especially when it is about new software components which need additional hardware components, a lot of working hours or, even worst, more actions from other teams who also do not have the necessary resources. The second ProgressM principle also comes from practice. It was found that essential failure 
factors, especially in large scale projects, are coming from activities that are not suitable treated in the project. Because of the lack of knowledge needed for a specific part of the project, that activity gets not enough attention. For example, a new original idea or technique must be implemented. For a new idea, the way to do it is unknown. The manager asks to be done. He pushes the developer's team to make that component without any plan. Is a new idea, we have no plan, just do it. Is that so? Can be done without the proper knowledge? The developing team tries to understand and to build a solution, but they are only developers. They are not mathematicians, physicians, economist to split the process into significant parts. After considerable effort, the developing team will fail. In ProgressM the project manager must assure that all activities are divided into small components with a theoretical solution which will be the base for the developing team. The second ProgressM principle starts from the idea that any activity can be divided into small and multiple steps that must be completed into a consecutive sequence in order to assure the progress. With this approach, the project will get a significant success probability. Also, the deciding factor in the project must assure all the small steps needed for the entire project. If the manager has not enough knowledge, he will ask for help from different external resources. With this principle, no ambiguity will remain, and no team participant will have to do something not fitted with his knowledge. The second principle is also applied for the global progressive steps of a large project. It means small targets must be planned for the whole project progress. If a large target is adopted, it must be divided into small parts in order to fix and get the progress in multiple small steps. What means large and small progress steps it is, of course, a relative criterion. For example, BIDS with no real-time stream analysis cannot be transformed over the night into real-time BIDS. The progress must be divided into several parts and implemented step by step, especially when it is about a new or an unknown activity. First, it will be inserted the real-time stream ETL processes, the database architecture will get new features, the server architecture will be modified because of new computational needs, the data warehouse will be reconfigured, new real-time data transformation processes will be implemented, the processes and services of the real-time event will get born, real-time automated decisions can be implemented and on end the real-time reports can be built and delivered. The third principle asks for a key factor in order to quantify the evolution of the project activities during the time. Each component will have a defined key factor. This measure will indicate if the activity goes on the right way, if the state of the project is improving or if additional actions must be made in order to help the development into a specific part of the project. It was found, especially in agile software development, because of a significant flexibility level adopted into the team, the project is not evaluated for an essential time period just because there is no key factor measured. Anyone works outside of a realistic plan, and after an important time elapsed they meet again, and they find there is no progress in the last days or weeks. This is why the ProgressM methodology asks for a key factor to be measured in any activity in order to have a measurable point of view for each activity. The idea is simple to be implemented; the key factor can be a number or even a Boolean variable for small processes. For more aggregated processes the key factor can be the time-delay inserted into a time-chain, an expenditure cost which can be optimized and so on. The ProgressM methodology organizes the project management in order to optimize the activity and resources, divide the whole process into small parts and measure the efficiency of each activity in order to progress. The methodology can include any agile, iterative, incremental or fixed plan models for each project components. The ProgressM methodology can be applied to small or large scale projects, in small or large teams and will cover the main failure mistakes in order to increase the success factor. 
www.conferenceie.ase.ro

\section{Conclusions}

The new economic demands require the use of real-time informatics systems. Large volumes of data in real-time streams and managed in order to find patterns and to build events and decisions based on the current data values. The low latency decisions are automatically assembled and executed in order to obtain a small delay. The automated decision processing is a significant part of the decision tree in the modern BIDS. The complexity of the real-time BIDS decision systems asks for an appropriate methodology in order to manage the BIDS project to design, implement, maintain and improve the system. Specially designed for realtime projects, the ProgressM methodology offers multiple advantages. It combines the flexibility of the agile methods with the iterative steps needed in order to achieve a demanded performance level. The connections between the human factor and the automated decision level are essential parts of the BIDS functionality. The Progressive Management Methodology permits progress steps to be made in order to improve the entire BIDS. To improve the functionality and the results obtained, after the first phase of implementation, new requirements are born from the missing points of the current version. Once new resources are available, a new progressive step can be initiated. The ProgressM model integrates the concept of the necessary and sufficient resource, the small, multiple, complete and consecutive steps concept together with the key factor control steps concept. All of these will contribute to a stable, organized and flexible project management methodology for any real-time BIDS. The ProgressM methodology has no limitation; it permits large scale projects to be implemented with controllable costs and resources.

\section{Acknowledgement}

This paper was co-financed by Algorithm Invest SRL (https://algoinvest.biz).

\section{References}

[1] Gartner, Inc. Gartner Says Worldwide Business Intelligence and Analytics Market to Reach \$18.3 Billion in 2017. Sydney, Australia, 2017. Available at: https://www.gartner.com

[2] Gartner, Inc. Gartner Says Business Intelligence and Analytics Leaders Must Focus on Mindsets and Culture to Kick Start Advanced Analytics. Egham, UK, 2015. Available at: https://www.gartner.com/newsroom/id/3130017

[3] H. J. Watson. Tutorial Business Intelligence - Past, Presence and Future. US: Communication of the Association for Information Systems, Volume 25, Article 39, 2009. Available at: http://aisel.aisnet.org/cais/vol25/issl/39

[4] R. F. Bales. Interaction process analysis: A method for the study of small groups. Reprint at Chicago, University of Chicago Press, 1950 republished in 1976. ISBN: 9780226036182

[5] D. J. Power. A Brief History of Decision Support Systems. DSSResources.com, World Wide Web, 2003. Available at: http://DSSResources.COM/history/dsshistory.html

[6] R. H. Jr. Sprague, H. J. Watson. Bit by Bit: Toward Decision Support Systems, US: California Management Review, vol. XXII, no. 1, 1979.

[7] S. Y. Hung. Expert versus novice use of the executive support systems: an empirical study. Maui, Hawaii, US: Proceedings of the 34th Annual Hawaii International Conference on System, 2001. DOI: 10.1109/HICSS.2001.927187

[8] BI. Wikipedia Online Encyclopedia. Business Intelligence online presentation. 2018. Available at: https://en.wikipedia.org/wiki/Business_intelligence

[9] Y. Riahi. Business Intelligence: A Strategy for Business Development. SSRG International Journal of Economics and Management Studies. Volume 4, Issue 9, 2017.

[10] L. Columbus. The state of business Intelligence. Forbes online, 2018. Available at: https:// forbes.com/sites/louiscolumbus/2018/06/08/the-state-of-business-intelligence-2018 
www.conferenceie.ase.ro

[11] D. E. Avison, G. Fitzgerald. Information systems development, in Rethinking Management Information Systems: an Interdisciplinary Perspective, Oxford, UK: Oxford University Press. W. Currie and B. Galliers edition, pp. 136-155, 1999.

[12] J. L. Wynekoop, N. L. Russo. Studying System Development Methodologies: An Examination of Research Methods. Information Systems Journal, (7), 1997, pp. 47-65.

[13] N. L. Russo, E. Stolterman. Exploring the assumptions underlying information systems methodologies, Information Technology \& People, 13:4, 313-327, 2000. ISSN: 0959-3845

[14] E. Stolterman. How system designers think about design and methods: some reflections based on an interview study, Scandinavian Journal of Information Systems, 4, 137-150, 1992.

[15] M. Shaw. Prospects for an engineering discipline of software, IEEE Software, 7, 1990.

[16] C. R. Necco, C. L. Gordon, N. W. Tsai. Systems Analysis and Design: Current Practices, Management Information Systems Research Center, University of Minnesota, Quarterly, Volume 11. No. 4, pp. 461-476, 1987. DOI: $10.2307 / 248975$

[17] S. M. Dekleva. The influence of the information systems development approach. Management Information Systems Research Center. University of Minnesota. Quarterly, Volume 16, No. 3, pp. 355-372, 1992. DOI: 10.2307/249533

[18] J. P. Bansler, K. Bodker. A reappraisal of Structured Analysis: design in an organizational context, ACM Transactions on Information Systems, 11:2, 165-193, 1993.

[19] B. Fitzgerald. The use of systems development methodologies in practice: a field study, Information Systems Journal, 7, 201-212, 1997.

[20] C. J. Hardy, J. B. Thompson, H. M. Edwards. The use, limitations and customization of structured systems development methods in the United Kingdom, Information and Software Technology, 37:9, 467-477, 1995. DOI: 10.1016/0950-5849(95)97291-F

[21] L. T. Moss, S. Atre. Business Intelligence Roadmap: The Complete Project Lifecycle for DecisionSupport Applications, Boston, USA: Addison-Wesley Professional, 2003.

[22] L. T. Moss. Beware of Scrum Fanatics On DW/BI Projects. Enterprise Information Management Institute Magazine, Volume 3, Issue 3, 2009. Available at: http://www.eiminstitute.org/library/eimi-archives/volume-3-issue-3-march-2009-edition/ bewareof-scrum-fanatics-on-dw-bi -projects

[23] B. W. Boehm, R. Turner. Balancing Agility and Discipline: A Guide for the Perplexed, US: Addison-Wesley Longman Publishing Co., Inc., 2003. ISBN 0-321-18612-5

[24] J. Charvat. Project Management Methodologies: Selecting, Implementing, and Supporting Methodologies and Processes for Projects, Hoboken, NJ, US: Wiley\&Sons, 2003

[25] H. Wells. How Effective Are Project Management Methodologies? An Explorative Evaluation of Their Benefits in Practice. Project Management Journal, Vol. 6 Issue 43

[26] H. D. Benington. Production of Large Computer Programs. IEEE Annals of the History of Computing. IEEE Educational Activities Department. 5 (4): 350-361, 1983.

[27] H. Mills, M. Dyer, R. Linger. Cleanroom Software Engineering. IEEE Software. 4 (5): 19, 25, 1987. DOI:10.1109/MS.1987.231413.

[28] W. S. Humphrey. Characterizing the software process: A maturity framework. IEEE Software. 5 (2): 73-79, 1988. DOI:10.1109/52.2014.

[29] B. Boeh. A Spiral Model of Software Development and Enhancement, ACM SIGSOFT Software Engineering Notes, ACM, Volume 11, Issue 4, 1986. DOI: 10.1145/12944.12948

[30] P. Kruchten. The Rational Unified Process: An Introduction. US: Addison-Wesley. 1998

[31] K. Schwaber. Agile Project Management with Scrum. Microsoft Press, 2004.

[32] K. Beck. Extreme Programming Explained: Embrace Change. Addison-Wesley, 1999.

[33] K. Beck, M. Beedle, A. Benekum, A. Cockburn, W. Cunningham, M. Fowler, J. Grenning, J. Highsmith, A. Hunt, R. Joffries, J. Kern, B. Marick, R. C. Martin, S. Mellor, K. Schwaber, J. Sutherland, D. Thomas. Manifesto for Agile Software Development, 2001. Available at: http://agilemanifesto.org/principles.html 